\title{
Advances in Synthesis and Application of Nanometer Drug Carriers
}

\author{
Jianping Wu,Keming Wang, Yuquan Peng \\ School of Pharmaceutical Engineering Technology, Jiaxing University of Applied Science and Technology, Zhejiang, \\ China
}

\begin{abstract}
The main reason for the formation of nano-biotechnology is due to the penetration of nanotechnology in the biological field, nanotechnology research center is the study of nano-drug carrier. Nano-drug system targeted drug delivery to achieve drug release, increase the insoluble drugs and peptide drug bio-efficiency, reduce the toxicity and application of drugs and other aspects of the development of good prospects, and thus become one of the key research in recent years' field. Synthesis and application of nanometer drug carriers this review is presented in recent years and its application to provide a comprehensive basis for the treatment process. Describes the nature and preparation of nano-drug carrier methods, in recent years, people have been widely concerned by scholars. Compared with the nano-drug delivery, the general pharmaceutical cannot have to extend the role of drugs, strong efficacy, and the advantages of small drug response. Nano-materials, the specific surface area, surface activity, high catalytic efficiency, surface active center, adsorption capacity and other characteristics, which has many excellent features and new features.

KEYWORDS: nano drug carrier; nano biotechnology; nanoparticles
\end{abstract}

\section{Introduction}

The route of administration is the direction of the drug preparation. Oral administration is the key to the first effect. The first effect affects the role of drugs, such as proteins, peptide drugs and so on. The effects of drugs are usually targeted to human organs and tissue-specific specific structures such as human immune system, reticuloendothelial, and blood-brain barrier transport. On the one hand, the body's self-protection system is used for macromolecular drug effects to reach the lesion's ability. For example, a normal body, blood - brain barrier, the general monoamine in the central nervous system cannot pass. On the other hand, the drug cannot be applied directly, but must be carried out by certain reagents and mainly hinders the application of the drug in the drug with greater side effects and the physical properties of the drug itself (e.g. poorly soluble drugs). Overcoming the obstruction of the human health drug administration is the direction of modern medicine research to improve the physical properties of drugs (solubility, dissolution rate, etc.) to reduce the adverse effects of drugs, for enhanced performance of drugs, increased bioavailability, there are also ways to solve this problem by combining nanotechnology with biomedicine. Traditional pharmacy, pharmacology and other multidisciplinary and nano medical combination of modern nanotechnology products. According to the american health association, the use of nanotechnology to control drug efficacy, diagnosis, monitoring and nanotechnology of biological systems. Drug formulations that explore the transport and targeting characteristics of the theoretical formula and diagnostic mode of efficacy of the nano pharmaceutical problem at the forefront, mainly including precise and specific clinical pathology to determine the location of the environment associated with the lesion and at the same time The risk of adverse effects to select the appropriate nano-carrier to meet specific requirements. Therefore, they need to find a way to mass production of nano-drug convenience.

\section{Introduction}

\subsection{Objective and significance of nano-drug carrier research}

Nano-drug carriers are usually synthesized from natural or polymeric materials at a size of 10-1000 nm because of their targeted and slow release, so the carrier material has other significant advantages of biocompatible biodegradability, which has been widely used in pharmaceuticals preparation development. The nanoparticles are used as drug carriers in which the therapeutic drug is encapsulated into the nanoparticles or adsorbed on the surface thereof by means of

Copyright (C) 2018 -. This is an Open Access article distributed under the terms of the Creative Commons Attribution-NonCommercial 4.0 International License (http://creativecommons.org/licenses/by-nc/4.0/), permitting all non-commercial use, distribution, and reproduction in any medium, provided the original work is properly cited. 
targeted molecules, for efficient delivery and gene therapy. Nano-carrier technology is an important development of nano-biotechnology [1].

Nanotechnology and nanomaterials will be applied to the pharmaceutical field to form nano complex carriers, in vivo drugs that carry nanoparticles of drugs or embedded in their surface after adsorbing nanoparticles, to form a stable nano sized drug delivery system. Nano-carrier drug delivery methods are hydrophobic, electrostatic, hydrogen bonding, and covalent role. In the field of drug discovery. Which has the following advantages:

(A) Enhance the dissolution of insoluble drugs. The hydrophobic drug will be precipitated in the blood circulation and the drug is difficult to reach the lesion site. Therefore, the hydrophobic drug will be packaged in the nano-drug carrier, and the outer layer of the nano carrier is usually a hydrophilic layer, which can better avoid the drug aggregation and precipitation, thereby ultimately enhancing the solubility of the drug.

(B) To prevent drug degradation, improve the stability of the drug, for the biological activity of drugs, such as easily degraded in the human camptothecin, and therefore lose the drug effect. And the use of nano-drug carrier can be wrapped to achieve the purpose of avoiding its degradation.

(C) Improving the properties of the drug dispersion in the body to reduce or eliminate tissue damage caused by leakage of the resulting drug. After this, by the reticulate endothelial system of phagocytic nano into the human body, you can quickly phagocytic cells in the region. For example, doxorubicin can not only destroy cancer cells, but also greatly damage the normal cells, nano-drug carrier can focus on the lesion site (targeted release), so that can reduce the damage to normal tissue.

(D) Improving the absorption of drugs. Because the nano-drug carrier is too dispersed, the surface area and the surface has a special function, which can better improve the drug contact area and time, in the lesion, so that the efficiency of the drug can be improved.

(E) Membrane transduction mechanisms that enhance the permeability of drugs that are beneficial to some of the special parts of the biofilm.

\subsection{Types and characteristics of nano-drug carriers}

According to the preparation of nano-drug carrier material can be divided into nano-particles, nano-milk, nanomicelles, nano-suspension, and drug-like body [2].

The nanoparticle nanoparticles are solid colloidal particles smaller than the lttm polymer colloid drug delivery system [3]. Is a solid formed in the polymer backbone, in which the drug can be dissolved or coated on a solid adsorbent by preparing different drug cladding modules into nano spheres and nano capsules. When the drug is dissolved, dispersed or adsorbed, ball-forming polymer drugs are called nano spheres; nano capsules are spherical nanoparticles [4] formed by the encapsulation of solid or liquid drugs, wrapped by polymer films of natural or synthetic polymers.

Nano emulsion is also known as micro emulsion, particle size 10-100nm between, usually, nano-emulsion is composed of oil, water, surfactant and surfactant composition of the colloidal dispersion system, the general formation of spherical droplets can make The size of the more uniform, transparent or translucent, and have the thermodynamic stability, mild preparation conditions make nano-emulsion physical stability better, can enhance the stability of the drug; by dissolving the oil phase and surfactant chain, can improve the drug the solubility of drugs and promote drug absorption, improve drug utilization of nano emulsion is a new type of drug carrier [5].

Nano micelles can be prepared by block polymer nano-micelles as a drug carrier, self-assembled structure formed in water is amphiphilic block polymer dissolved synthesis, the particle size is generally $5 \mathrm{~nm}-100 \mathrm{~nm}$. Its advantages are: high drug dose, wide range, strong stability, the role of a long time, the unique distribution, high utilization, toxicity, is widely used as anti-cancer drugs, antibiotics, antihypertensive drugs and genes treatment of drugs and other hydrophobic drugs contained [6].

Nanoparticles Suspension nano-suspensions under certain conditions, special techniques and equipment are used to pulverize the drug to obtain nano-suspensions. In addition to increasing the adhesion and the crystalline structure of the amorphous particles compared to conventional dosage forms, it also increases the rate of dissolution of the poor solubility of the poorly soluble drug, especially for large doses of soluble or injectable poor absorption of oral medications. Such as the anti-AIDS drug bupravaquone in the mucosal adhesion of nano-suspension preparation, bioavailability of up to $40 \%$, and the effect is improved, and the dose is greatly reduced [7].

Drugs are formed by covalent bonds with lipids to form drug bodies, which are automatically formed due to changes in the solubility characteristics in the medium. Drug quality is a new type of drug delivery system; particle size range is generally 10-200nm. Drugs are nanoparticles, and they also overcome the shortcomings of drug leakage and skeleton instability, improve drug targeting and biocompatibility, and increase stability [8]. 
Nano-drug carrier types are rich and varied, more common with polymer micelles, liposomes nanoparticles, polymer-drug copolymers, ceramic nanoparticles, carbon nanotubes, etc., according to its characteristics are applied to different drug delivery system the nano-drug carrier structure.
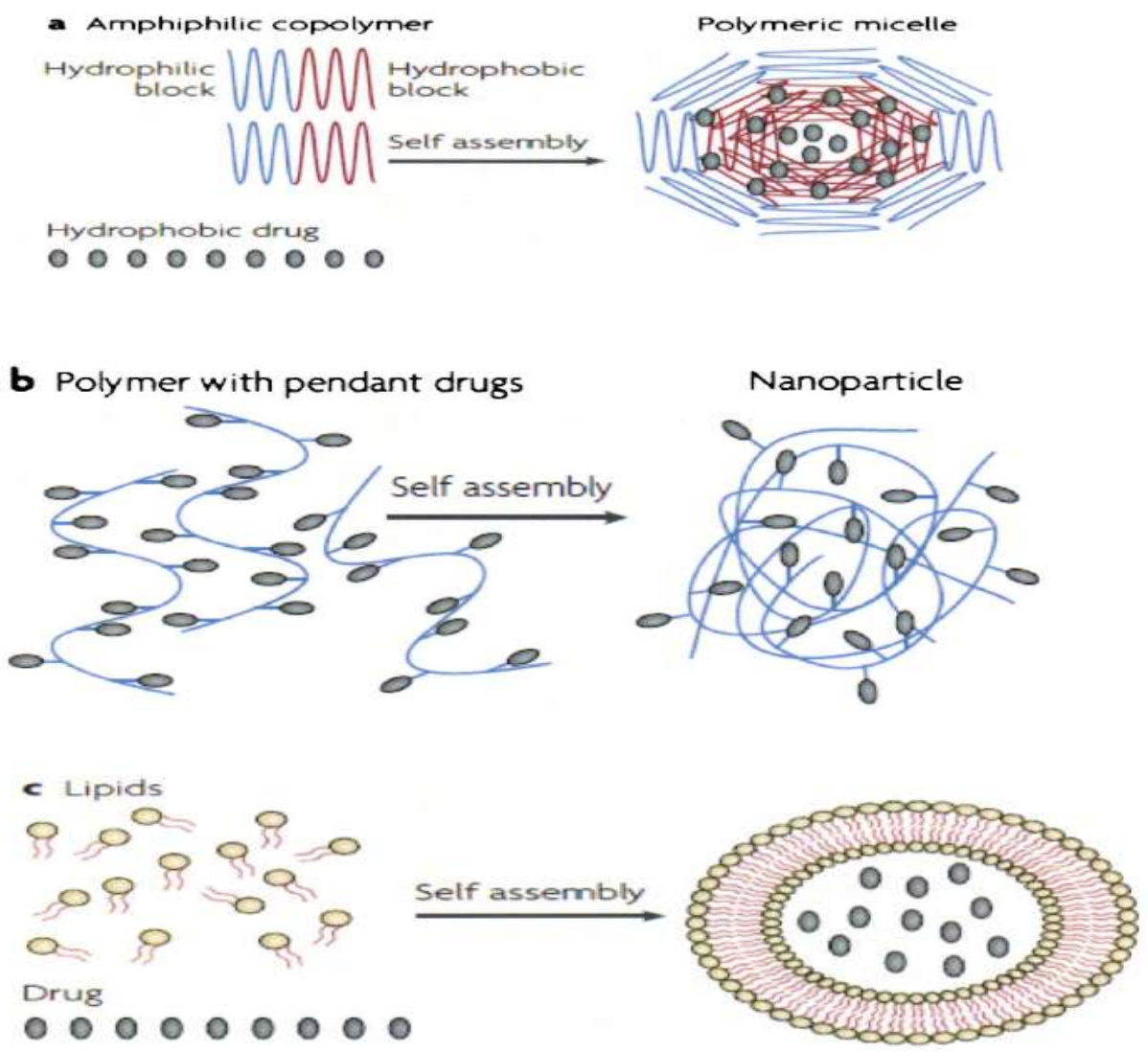

\subsection{Transport of nano-drug carriers}

In general, nanoparticles are engulfed by the reticular endothelial system during circulation, and the flow of nanoparticles is affected by their size and surface characteristics [9]. Diameter size can be adjusted to be one of the advantages of nanoparticles. But the particles cannot be too large, otherwise it is engulfed by the macrophages of the reticuloendothelial system. Particle size of 100-150nm nanoparticles for the most effective.

In addition to particle size, in the vascular circulation is also affected by the surface characteristics. Ideally, the nanoparticles will have a longer cycle time on the hydrophilic surface of the blood vessel. Can be obtained by two methods, namely, nanoparticles modified by hydrophilic polymers, or hydrophobic and hydrophobic copolymerization to form hydrophobic core, hydrophilic shell of nanoparticles. However, changing the surface charge of nanoparticles can affect their fate, for example, neutral nanoparticles are negligible compared to negative and positive nanoparticles when ingested cells are ingested.

Meet the above conditions, can better reach the recipient tissue. Binary complex in the drug transport target effect is not satisfactory, only on the macrophages are more concentrated organs have a certain effect [10]. But can be selected specific ligand or antibody linked to the binary match to overcome the above problems. Active targeting refers to a drug delivery system constructed by specifically interacting and enriching specific organs, tissues, or cells by means of certain specific biochemical environments in the organism, or by the antigen-antibody response mechanism of the organism. According to different mechanisms of action, active targeting is divided into antigen-antibody binding targeting and ligand-receptor binding targeting.

\section{Preparation methods of nanometer drug carriers}

\subsection{Liposomes}

The overall structure of the nano-drug carrier is liposomes, which are the earliest developed nano-drug carriers before, until now, liposomes have also improved and used in more disease treatments. Membrane Evaporation [11], 
reversed-phase evaporation [12], thin-film ultrasonic dispersion [13] and so on are the main methods for the synthesis of nano-liposomes.

Membrane evaporation method in the organic solvent to the drug and cholesterol, phospholipid compatibility, the liposomal suspension is the evaporation of the solvent so that drugs and phospholipids and other film-forming materials to form a symmetrical lipid film in the flask wall and added to the wash film rinse obtain. Lvwen li et al. Synthesized 50nm liposomes by combining thin film evaporation and freeze-drying with $187.5 \mathrm{mg}$ of phospholipid, cholesterol, 93.8 $\mathrm{mg}$ and $37.5 \mathrm{mg}$ lamp and $30 \mathrm{ml}$ of dichloromethane as the main raw material.

The aqueous solution of the drug is added to the mixed solution of lecithin, cholesterol and organic solvent to form a relatively stable CDE type emulsion. The organic solvent is removed and the phosphate buffer is added to hydrate and continue to short-term evaporate at low milky yellow liposomal suspension. L. Liu and other use of reverse phase evaporation with lecithin, cholesterol, streamside made of particle size of $562 \mathrm{~nm}$ coverage of $55 \%$ to $65 \%$ of the cationic liposomes.

Thin film ultrasonic dispersion method first synthesis of phospholipid bilayer, by ultrasonic hydration dispersed liposomes. Y. Gu, Y. Shi, who used emodin, cholesterol, phospholipids, chloroform prepared a particle size of about 25 nm liposomes.

\subsection{Emulsion method}

Nano-drug in the emulsion synthesis method known as the emulsion method. The small particles are obtained by nucleating the small droplets formed by the action of the surfactant dispersion and the external force. But also by nature in the emulsion polymerization self-assembled nuclei. The main methods: emulsion polymerization, emulsion selfassembly method and membrane emulsion interface polymerization method

Emulsion polymerization emulsion polymerization nucleation is a discontinuous process. Particle size obtained by emulsion polymerization varies in size, typically between $100 \mathrm{~nm}$ and several ums. C. Chauvierre, C. V authier, D. Labarre et al. [14] with dextran, dextran coated polyisobutylcyanoacrylate nanoparticles synthesized by anionic polymerization and two mechanisms of emulsion radical polymerization.

The self-assembly polymer nanocomposite has a core-shell structure by dissolving the amphiphilic polymer in an organic solvent and adding water to form an emulsion. The organic solvent is removed by vacuum distillation or solvent diffusion. Y. Li, S. Ikeda, K. Nakashima, H. Nakamura et al. Prepared polymethyl methacrylate micelles, and have a core-shell structure.

Membrane emulsification interface polymerization membrane emulsification interface is a special interfacial polymerization that occurs by the addition of an initiator to the uniform emulsion prepared in the nano porous membrane. Membrane emulsification can not only obtain a monodisperse, stable emulsion by a simple and effective method, but also an effective means of preparing functional microspheres and microcapsules.

\subsection{Micro emulsion method}

There is a fundamental difference between the micro emulsion and the emulsion. In the thermodynamic stability conditions can be spontaneously formed transparent or translucent micro emulsion dispersion system. But the effect is not stable, placed for a period of time will automatically stratify. The micro emulsion can form nano cells, and if the reaction is carried out in the reaction pool, the reaction product can be regarded as nano. Moreover, the micro emulsion reaction conditions are very mild, by changing the micro emulsion preparation method, the external conditions change more so that it changes, the small pool to change the shape, the product of the nano-structure is different. The most attractive is the micro emulsion, which can dissolve a large amount of water and a large amount of oil at the same time. It can make the above oil-soluble substances and water-soluble substances fully mixed, which greatly improves the efficiency of the reaction. At present, micro emulsion method has many people at home and abroad to study, according to the type of micro emulsion can be divided into normal phase micro emulsion $(\mathrm{O} / \mathrm{W})[15]$ and reverse micro emulsion $(\mathrm{W} / \mathrm{O})[16]$.

\subsection{Ultrasonic method}

Liquid phase internal molecules in the ultrasonic vibration occurs, the molecules change, when the intensity of ultrasound to a certain extent, will produce holes or cavitation bubble liquid molecules, this phenomenon is called ultrasonic cavitation technology [17]. Cavitation bubbles continue to produce and violate the collapse, when the cavitation bubble collapse, resulting in local high temperature and high pressure, temperature changes, and accompanied by a strong impact and a $400 \mathrm{~km}$ projection rate. Therefore, ultrasonic cavitation has a very special physical and chemical environment, through its conditions to achieve some special chemical reactions. For example, the phacoemulsification on a two-phase liquid interface can cause the reaction even in the absence of a catalyst. Now a lot of polymerization 
reactions are used in ultrasonic technology. By exploring the preparation of drug carriers under ultrasonic conditions, many methods have been improved and a better performance nanometer drug carrier has been prepared.

\subsection{Dialysis and solvent evaporation method}

The drug carrier is dissolved, the resulting solution is dispersed, evaporated or dialyzed into an organic solvent, and the drug or carrier is nucleated and aggregated due to drastically reduced solubility. Z. Yan et al. The preparation of methoxypolyethylene glycol-polylactic acid copolymer nanoparticles by phase separation/dialysis method and the study on the distribution of drugs in the nose. (TP-PLA-NP), TP-PLA-the average particle size of NP was determined by dynamic laser particle size analyzer $(167 \mathrm{~nm})$. The results showed that TP-PLA-NP as a nano-carrier can significantly reduce the toxicity of Tripterygium wilfordii.

\subsection{Precipitation method}

The product is precipitated in solution by chemical reaction, and the size of nano-drug particle size is affected by the reaction conditions. Y. Wu and so on in the preparation of nano-magnetic materials used in the method. Pillar calvo [18] used an interfacial deposition method to prepare chitosan and polyline-coated nano capsules to study the efficacy of capsules as indomethacin. The study found that the efficacy of coated chitosan increased by a factor of two, while the efficacy of coated with polyline did not change.

\section{Application and prospect of nanometer drug carrier}

\subsection{Application of nanometer drug carrier in clinical medicine}

Oral Drug Carriers: Drugs and Drugs are affected by the route of administration. Oral efficacy is mainly affected by two primary effects, biogenic enzymes and liver enzymes on the gastrointestinal tract that are epithelial cells. Many drugs are mostly due to the first effect and metabolic failure, such as peptides, protein drugs have not been a good therapeutic effect, oral route of administration usually must be changed to intravenous injection. As the non-targeted drug through the injection pathway steps can be evenly distributed in the systemic blood circulation, and then reach the lesion tissue, protein binding, excretion, degradation, etc., only a small amount of drugs to reach the lesion. The purpose of targeted drug delivery is to improve the drug concentration in the target area and improve the efficiency of the drug to reduce the side effects of drugs in the field has been an important research topic, nano-drug carrier research effectively solves these problems. After some oral medication in the nanoparticle wrapped under the gastric acid, pepsin on the drug decomposition decreased. In addition, the nanoparticles can easily promote the delivery of drugs with poor oral absorption specificity in the human body, prolonging the action time of the drug, and cyclosporine is usually used as an anti-rejection drug in clinical organ transplantation, and the water- Slow absorption, oral use of low bioavailability.

Anti-tumor drug carrier: In recent years, nano-capsule for anti-cancer drug carrier particles research has become the focus of attention of medical workers. Most of the anti-cancer drugs, in the cancer treatment of specificity is poor, the key is how to target the drug delivery to the tumor cells without damage to normal cells. Liposomes or microcapsules as a drug carrier can improve the water solubility of drugs, so that drug targeting, reduce drug toxicity and prolong the residence time, prolonged the survival time of tumor animals. Soma et al. [19] explored the effects of macrophages on the presence of doxorubicin-containing nanoparticles, and the results showed that cancer cells with nanoparticles containing doxorubicin toxicity were much more toxic than free doxorubicin. As a new anti-cancer drug paclitaxel encapsulated in PVP nanoparticles in vivo experiments, the tumor size of tumor-bearing mice was reduced and the survival time was evaluated for efficacy. The results showed that paclitaxel was less than the free paclitaxel nanoparticles the efficacy of the concentration was significantly increased. The experiment also prepared a package of PLGA nanoparticles targeting drug paclitaxel, as well as linked to a specific targeting malignant melanoma, breast cancer, mouse-derived anti-tumor-related antigen monoclonal antibody SM5-1 single chain antibody, the results show that it can be designed to kill liver cancer cells [20].

Targeting the carrier of the brain: the presence of the blood-brain barrier, the usual drug is not easy to enter the brain, so the part of the diagnosis and treatment is very difficult. Studies have shown that modified nanoparticles can pass through the blood-brain barrier, which acts as a drug delivery to the central nervous system. Camptothecin solid lipid nanoparticles were injected into mice and found that camptothecin was enriched in rat brain, liver, heart, spleen, brain AUC/dose and MRT increased by 10.4 and 4 times, so this nano granules can pass the blood-brain barrier, which is of special significance for the treatment of brain tumors [21]. Vector is a gene delivery: Antisense oligonucleotide technology is one of the gene therapy methods aimed at designing antisense nucleic acids against a specific target sequence based on the principle of nucleic acid hybridization, thereby inhibiting the expression of a particular gene. Antisense oligonucleotide drugs can hybridize to a particular target gene and interfere with the production of a pathogenic protein at the gene level. As the exonuclease and endonuclease of the in vivo are ubiquitous and degrading, 
the bioavailability of oligonucleotides is reduced. The use of nanotechnology gene transfer can overcome this shortcoming; nano-drugs have more than other carriers do not have many advantages in clinical applications has a very good prospect.

\subsection{Application of nano-drug carrier in pharmaceutical}

The drug-loaded nanoparticles are nanotechnology combined with modern medical products and are a super-ball drug carrier. In recent years, it is a new drug delivery and sustained release dosage form, including nanoparticles and nano capsules. Its advantage is smaller than the cells (10-1OOnm), so it can be absorbed through the organization and cells, after special treatment can be guided to the organization and management of the organ.

Controlled release of drug particles: with the previous control of the preparation of different formulations, the release of drug-loaded nanoparticles has a specific law of the process, due to the dissolution of the wall and the role of enzyme micro-organisms, drugs can make nanoparticles in the local stay and to achieve effective concentration, without causing systemic toxicity. For some of the immune system and the central nervous system drugs, the disease of the treatment of chronic diseases, long-term medication. Drug-loaded nanoparticles as a new type of drug-controlled release formulations, especially for these drugs. It was found that rats were subcutaneously injected with insulin nanoparticles. The hypoglycemic effect could last for three days and had a significant reaction with the absorption of the drug. It was also found that the hypoglycemic effect of insulin nanoparticles was almost three times a day with conventional insulin therapy have the same effect, thus proving that insulin can be made under the nano-carrier particles can prolong the role of time and lower blood sugar, more effective than the same dose of insulin.

Drug-loaded nano-enhancing drug targeting effect: such micro particles are prepared by the use of various human tissues or vectors of different affinity for the preparation of drug-loaded micro particles according to clinical needs, or the monoclonal antibodies are bound to the vector, the drug can be highly selective The dispersion in the role of the object, thereby enhancing efficacy and reduce side effects. From target organs, target cells to the most advanced intracellular target mechanisms, these three targeted therapy can be done through drug nanoparticles. When the drugloaded nanoparticles and the drug form a complex, depending on the purpose of the treatment, the choice of blood through the circulation or different ways to selectively enter the body's specific tissue and cells to achieve treatment. Characteristics of nanoparticles into the cell structure and to achieve gene therapy. Now the study of more objects with liver, blood system, gastrointestinal tract, lung, etc. in the liver, etc., for example, drug nanoparticle composite material in two ways to achieve positioning: the complex is kuepfer cell phagocytosis, in the liver drug accumulation, and then gradually fall into the blood circulation, so that the increase in liver drug concentration, reduce other organs side effects, known as passive targeting side effects; when the nanoparticles are small enough (100-150 nm), and covered with a special coating layer surface, kuepfer cells can escape phagocytic cells, connect monoclonal antibodies and other positioning in the role of liver parenchymal cells, known as active targeting [22]. Regardless of the approach taken, the size and shape of the particles is critical to achieving targeting in order to achieve localization while the specificity of the antibody on the cell surface can improve the selectivity.

Manufacturing nano-robots to replace traditional drugs: nano-robot, also known as molecular robots, is a combination of nano-mechanical devices and biological systems (such as enzyme and nano-gear combination). Nano-robot with its unique structure and nature, so that in many areas have shown extraordinary vitality, to solve the traditional drug mouth problems [23]. In the field of cardiovascular, nano-robot can be injected into the body, a blood vessel operation of the robot. These molecular robots capture energy in blood glucose and oxygen to remove blood vessel thrombosis, remove heart and internal arterial lipid deposits; in genetic engineering, gene assembly can be performed by nano robots, that is, the gene can be removed or harmful DNA, or in the normal DNA assembly into the chromosome, so that the normal operation of the human body; nano-robot can be used for organ repair, such as repair damaged tissue and organs, complete cosmetic surgery; In addition, nano-robot also swallowed bacteria and kill cancer cells in the body to monitor the disease.

\section{Conclusion}

The direction of exploration of nano-drug carriers is to be intelligent, the study of the preparation of nano-carrier and a specific drug combination to get with automatic targeting and quantitative timing of the release of nano-intelligent drugs to address the diagnosis and treatment of major diseases.

The application of nanotechnology in the field of biopharmaceuticals is one of the most promising research directions for nanotechnology in the 21 st century. The use of nano-drug carriers makes some of the better efficacy but difficult to dissolve, slow to dissolve, highly toxic, easily degradable in the body of the drug has been applied to find a new drug dosage form of excellent performance, its significance is no less than found new drugs. Now, there are several kinds of nano-drugs into commercial production, not only to human disease to bring a new gospel, but also created a considerable economic benefits. But the development of nano-drugs has many problems to be solved, such as nanodrugs into the human body, in the human body under complex biological environment, drug distribution, degradation 
and drug release process, the mechanism of drug action, drug on human function, metabolism, structure and other issues such as the impact of research is not thorough enough. The current research and development trends are mainly focused on the following aspects: finding new methods for the preparation of nano-materials; synthesizing and discovering new non-toxic or micro-toxic, biocompatible and biodegradable nano-drug carriers; to improve the targeting and sustainedrelease properties, stability, drug loading, and gradually realize the intelligence of the drug; study the mechanism of drug action, the development of nano-drug carrier in vitro and in vivo detection technology.

\section{References}

1. $\mathrm{Lu}$ W, i ZhangY, TanYZ, et a1. Cationic albumin-conjugated Pegylated nanoparticles as novel drug carrier forbrain delivery [J]. J Controlled Release, 2005, 107 (3): 428.

2. CX. Yi, JN. Yu, XM. Xu. Nano drug carrier in the development of traditional Chinese medicine Jing of the application [J]. Chinese Journal of Traditional Chinese Medicine, 2008,33 (16): 1936-1940.

3. Kreuter J, Hekmatara T, Dreis S, et al. Covalent attachment of Apo lipoprotein A-I and Apo lipoprotein B-1OO to albumin nanoparticles enables drug transport into the brain 1-J]. J Controlled Release, 2007, 118 (1): 54.

4. Yang, WL. Lu, Q. Zhang. Advances in Liposomal and Nanoparticle Drug Delivery Systems EJ3. Chinese Journal of Medical Science, 2006,28 (4): 583-589.

5. BP. Yang, WQ. Ouyang, XJ. Wu. Wait. The quality evaluation of resveratrol Nano emulsion [J]. Journal of Northwest Agricultural Sciences 2008,17 (2): 20-23.

6. HY. Lin, XY. Lu, N. Tang, et al. Preparation of Vincristine PEGPE Micelles and its inhibition on breast cancer cell growth [J3]. Biochemistry and Biophysical Advances, 2006, 33 (8): 769-774.

7. Jacobs C, Kayser O, Muller RH. Production and characterization of mucoadhesive nano suspensions for the formulation of bupravaquone [J]. Int J Pharm, 2001, 214: 3.

8. Guo, SQ. Deng. Application of nanotechnology in pharmacy research progress. Foreign Medical Antibiotics Volume, 2004,25 (5); 233-237.

9. FangC, ShiB, PeiYY, etal. In vivo tumor targeting of tumor necrosis factor2 alpha2 loaded stealth nanoparticles: effect of Me2PEG molecular weight aD particle size [J]. Eur J Pharm Sci, 2006,27 (1): 27236

10. Guangdong Medicine, 2009,30 (1): 17219 (in Chinese with English abstract) [J].

11. WL. Lu, JX. Guo, QN. Ping. Preparation and quality of breviscapine liposomes [J]. Chinese Journal of Natural Medicines, 2004, 2, (5)

12. Y. Gu, Y. Shi, SQ. Zhang, et al. Preparation and quality evaluation of emodinnano-liposomes [J]. Journal of the Fourth Military Medical University, 2003, 24 (5).

13. C hauvierre $\mathrm{C}, \mathrm{V}$ authier $\mathrm{C}$, Labarre Detal. Evaluation of the surface properties of dextran - coated poly (Isobutylcyanoacry late) nanoparticles by spin-labelling coupled with electron resonance spectroscopy [J]. Colloid Polym Sci, 2004, 282: 1016 -1025 .

14. L. Wang. Reverse micro emulsion polymerization and preparation of magnetic polymer nanoparticles [D]. Shanghai: Fudan University, 2001.

15. YQ. Liao. Methyl methacrylate emulsion and micro emulsion ultrasonic irradiation initiation polymerization and synthesis with inorganic nanoparticles [D]. Chengdu: Sichuan University, 2001.

16. CX. Li, ZH. Wang. Application of ultrasonic technology in preparation of nanomaterials [J]. Chemical Bulletin, 2001, 5: 268 271.

17. Pilar Calvo, Jose' L. Vidla-Jato, Maria JALonso. Evaluation of cationic polymer-coated nanocapsules as ocular drug carriers [J]. International Journal of Pharmaceutics, 1997, 53: 41 -50.

18. Soma CE, Dubernet C, Baratt G. Ability of doxorubicin-loaded nanoparticles to overcome multidrug resistance of tumor cells after their capture by macrophages. Pharm Research. 1999; 16 (11): 1710.

19. Kou G, Gao J. Wang H. Preparation and characterization of paditaxel-loaded PLGA nanoparticles coated with cationic SM5-1 single-chain antibody. J Biochem Mol Biol. 2007; 40 (5): 731-739.

20. SC. Yang, JW. Zhu, BW. Liang, et al. Camptothecin solid lipid nanoparticles [J]. Chinese Journal of Pharmacology, 1999,34 (2): 146-150.

21. Yue G Z, Qiu Q, Gao B, et a1. Generation of continuous and pulsed diagnostic imaging X-ray radiation using a carbonnanotube-based field-emission cathode [J]. Appl Phys Lett, 2002, 81: 355

22. DZ. Liu. Modern ray detection technology. Beijing: China Standard Press, 999.42

23. Helga E. De Vries et al. Blood-brain barrier in neuroinf lammatorydiseases [J]. Pharmacol Rev, 1997, 49: 143-155

24. S. E. Gelperina, Z. Smmirnova, A.S. Khalanskiy, IN. Skidan, AIBobruskin, J. Kreuter. Chemotherapy of brain tumours using doxoru bicin bound to polysorbate 80-coated nanoparticles. Proceedings of the 3rd World Meeting APV / APGI, Berlin, 2000, 3/6th April 2000, 441-442

25. D. J. Selkoe. Alzheimer's disease: genes, proteins and therapy. Phys Rev. 2001, 81: 741-766 